\title{
Buckyballs
}

\section{ELECTRONIC STRUCTURE AND SUPERCONDUCTIVITY}

\author{
J.L. Martins \\ Department of Chemical Engineering and Materials Science \\ University of Minnesota, Minneapolis, USA
}

\begin{abstract}
Electron-phonon coupling may be responsible for superconductivity in alkali metal interstitial alloys based on "buckyball", or buckminsterfullerene $\mathrm{C}_{60}$, the almost spherical carbon molecule, the third known form of crystalline carbon found recently in macroscopic quantities in graphite soot.
\end{abstract}

Perfect geometrical shapes with high symmetries have always been found fascinating. The synthesis in 1990 of crystals containing cabon-60, a molecule with icosahedral symmetry where all the 60 carbon atoms are equivalent, generated excitement in the physics, chemistry and materials science communities. The study of these crystals has yielded surprising discoveries including a new family of superconductors with high critical temperatures $T_{c}$, notably $T_{c}=31.3 \mathrm{~K}$ for $\mathrm{Rb}_{2} 2$ $\mathrm{CsC}_{60}$. We shall review briefly the electronic properties of $\mathrm{C}_{60}$ and its compounds.

The truncated icosahedron shape for $\mathrm{C}_{60}$ was first postulated by R. Smalley's group in 1985. They observed that carbon clusters with 60 atoms were uniquely stable and abundant in molecular beams generated with a laser vaporization source. Searching for an explanation for this unusual stability, they found the 60 -atom structure shown in Fig. 1. This structure is unique in its high symmetry and satisfies the basic rules of carbon chemistry. The twelve pentagons and twenty hexagons of the truncated icosahedron are familiar to most of us as the shape of a football. This isomer of $\mathrm{C}_{60}$ was named "buckminsterfullerene" after the architect Buckminster Fuller who designed geodesic domes with icosahedral symmetry. Owing to its almost spherical shape, the molecule is familiarly called a "buckyball".

Subsequent molecular beam experiments have confirmed the cage structure, but the icosahedral symmetry of $C_{60}$ was only established after Krätschmer,

Professor José Luís Martins received his Ph.D. in physics from the Ecole Polytechnique Fédérale de Lausanne, Switzerland. He was a postdoc at the Solar Energy Research Institute in Golden, Colorado, USA, and a Miller postdoctoral fellow in the Physics Department, University of California, Berkeley, USA. He is the Ray K. Johnson Professor in the Department of Chemical Engineering and Materials Science, University of Minnesota, Minneapolis, MN 55455 , USA. His present address is: Departamento de Física, Instituto Superior Técnico, Av. Rovísco Pais 1, P-1096 Lisbon, Portugal.
Huffman, and co-workers produced macroscopic quantities using an electric arc discharge between two graphite rods. With solid $\mathrm{C}_{60}$ ("'fullerite") available, the nuclear magnetic resonance (NMR), infrared, Raman, X-ray diffraction, and photoelectron spectra were soon measured, and all showed characteristics of the highly symmetric shape of a truncated icosahedron. For example, the NMR signal contains a single line, demonstrating that all 60 atoms occupy equivalent sites in the molecule.

\section{Crystalline Carbon}

Carbon's ability to form covalent bonds from atomic configurations with different degrees of s-p hybridization is responsible for the enormous variety of organic and biological molecules. Yet, pure carbon was thought to occur in only two crystalline forms: graphite and diamond. Graphite, which has $\mathrm{sp}^{2}$ hybridization, is a layered compound (Fig. 1): each graphite layer has a hexagonal honeycomb structure with short, strong bonds between carbon atoms. The interlayer distance is large and the interaction between the layers is weak. Diamond (and its hexagonal variety lonsdaleite) has $\mathrm{sp}^{3}$ hybridization and forms a tetrahedral network of strong bonds (Fig. 1).

$\mathrm{C}_{60}$-fullerite is a new, third form of crystalline carbon. The $\mathrm{C}_{60}$ molecule resembles a graphite layer wrapped around a sphere with twelve pentagonal "defects". These are required by geometrical constraints to close a hexagonal graphitic network onto itself. The bond lengths in $C_{60}$ are $1.40 \AA$ and $1.46 \AA$, and the separation between "balls" is $3 \AA$. These values are close to the $1.42 \AA$ intralayer bond length and $3.35 \AA$ interlayer separation in graphite. The curvature of the bonding network mixes some $\mathrm{sp}^{3}$ character to the mostly $\mathrm{sp}^{2}$ atomic hybridization in the $\mathrm{C}_{60}$ molecule. Three of the four valence electrons of each carbon atom are shared in the $90 \sigma$ bonds that are along the edges of the truncated icosahedron. The remaining valence electron is in the $\pi$ orbitals. These are formed from radially oriented atomic orbitals that are mostly $p$ with $\approx 8 \%$ mixing of $\mathrm{s}$ character. The $\pi$ bonds are delocalized (aromatic), although they are somewhat stronger in the hexagon-shared edges of the truncated icosahedron.

The $\mathrm{C}_{60}$ molecule has a quasi-spherical shape with a cage diameter of $7 \AA$. Its "surface" should be "slippery" and unreactive like graphitic sheets. Solid $C_{60}$ has a cubic dense packing of molecules, with a $10 \AA$ separation between molecular centres. The orientation of the molecules in the crystalline phase is one of the interesting aspects of $\mathrm{C}_{60}$, because the weak interaction between molecules means that very small energy barriers exist between a large number of symmetrically equivalent orientations. Thermal analysis shows two phase transitions at $\approx 220 \mathrm{~K}$ and $\approx 250 \mathrm{~K}$. The high temperature phase has a face-centered cubic ( $\mathrm{fcc}$ ) lattice and is orientationally disordered. The low temperature phase has a simple cubic lattice and is an orientationally ordered superstructure of the high temperature phase. "Jumps" between molecular orientations
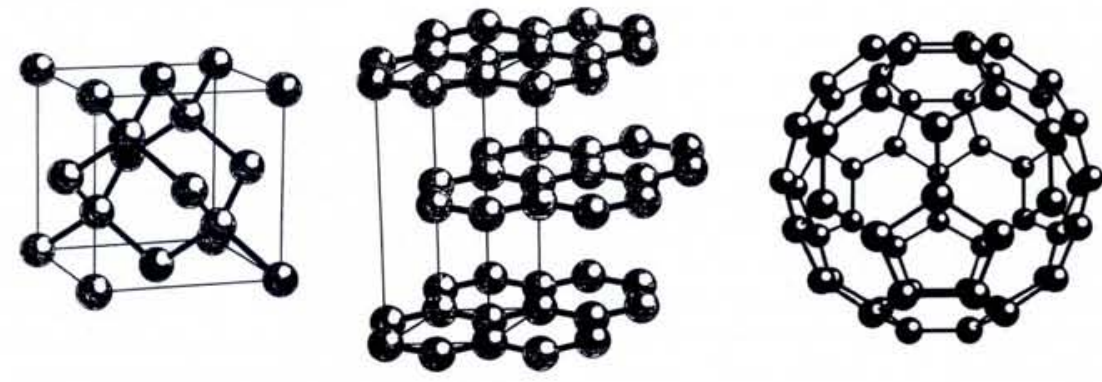

Fig.1 - Basic structures of carbon crystals: from the left, the tetrahedral network of diamond, the hexagonal layers of graphite, and the buckyballs of fullerite. 
have been studied as a function of temperature by NMR and neutron diffraction. The "rotational" motion of the $\mathrm{C}_{60}$ molecules in the crystalline phase is one of the interesting physical properties of fullerite.

\section{Electronic Spectrum}

The electronic spectrum of $C_{60}$ has been observed by photoemission and inverse photoemission spectrometry for occupied and empty states, respectively. The calculated density of states is in excellent agreement with the experimental spectra (Fig. 2). The sharp spectral features in Fig. 2 are due to the high degeneracy of the molecular states and the small band dispersion in fullerite. Neither diamond nor graphite have such a large number of sharp spectral features.

The electronic structure of $\mathrm{C}_{60}$ can be simply understood if we use the quasispherical shape and high symmetry of the molecule. Fig. 3a shows a contour plot of the amplitude of the wavefunction of the lowest unoccupied state of $\mathrm{C}_{60}$ on a surface of a sphere with $3.2 \AA$ radius. The polar coordinates of the figure are similar to latitude and longitude in world maps: the contour lines are the equivalent of altitude in geographical maps. Fig. 3b shows a contour plot of a combination of spherical harmonics with angular momentum $I=5$ that belong to $\mathrm{a}_{1 u}$ representation of the $I_{k}$ icosahedral point group. The similarity between the wavefunction and the spherical harmonic is striking, particularly if we look at the the topology of the nodes of the two functions.

The molecular wavefunction shown in Fig. 3a is important in understanding superconductivity in the alkali fullerides because their conduction bands are derived from that molecular state. The symmetry of the three-fold degenerate state is $\mathrm{t}_{1 u}$ in the $\mathrm{I}_{h}$ icosahedral point group. We can recognize in Fig. $3 a$ that the wavefunction has a ring-like pattern around the "equator" where the three degenerate $\mathrm{t}_{1 u}$ wavefunctions correspond to three mutually perpendicular "rings". This $t_{1 u}$ state is a $\pi$ orbital and is related to an $I=$ 5 spherical harmonic: we shall call it a $\pi_{5}-\mathrm{t}_{1 u}$ orbital.

\section{Alkali Fullerides}

All the alkali elements form interstitial alloys with solid $C_{60}$. We find again a similarity with graphite which forms intercalated compounds where the alkali atoms are sandwiched between the graphite layers. The highest alkali (A) concentration is achieved for $A_{6} C_{60}$. The crystal structure of $\mathrm{K}_{6} \mathrm{C}_{60}, \mathrm{Rb}_{6} \mathrm{C}_{60}$ and $\mathrm{Cs}_{6} \mathrm{C}_{60}$ is a body-centered cubic (bcc) packing of $\mathrm{C}_{60}$ molecules with the alkali atoms occupying all the quasi-tetrahedral interstitial sites. The bonding between the alkali atoms and $\mathrm{C}_{60}$ is ionic, with the alkali valence electron transferred to the

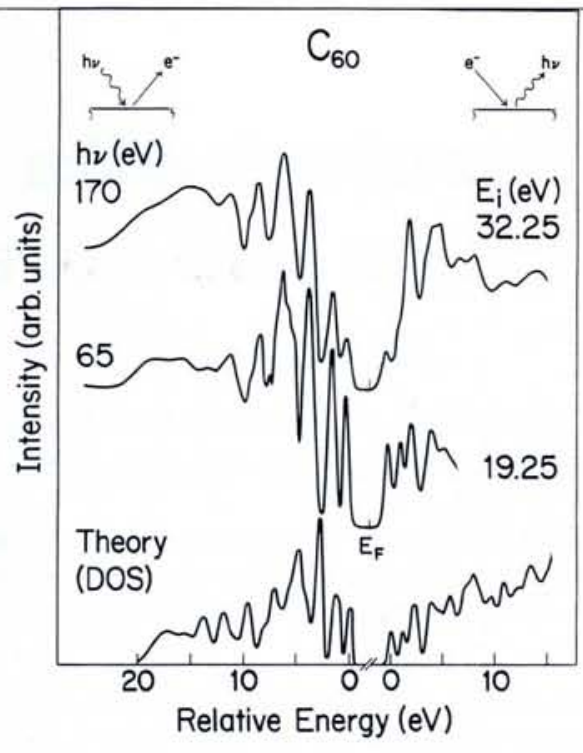

Fig. 2-Calculated density of states (DOS) compared with the measured photoemission and inverse photoemission spectra. The DOS curve shown in the lower part was Gaussian broadened. The photoemisson energy distribution curves are shown in the left of the upper part for two different incident photon energies $\hbar \omega$. The inverse photoemission photon distribution curves are shown on the right of the upper part for two different incident electron energies $E_{i}$. The photoemission and inverse photoemission processes are sketched on the top of the figure.
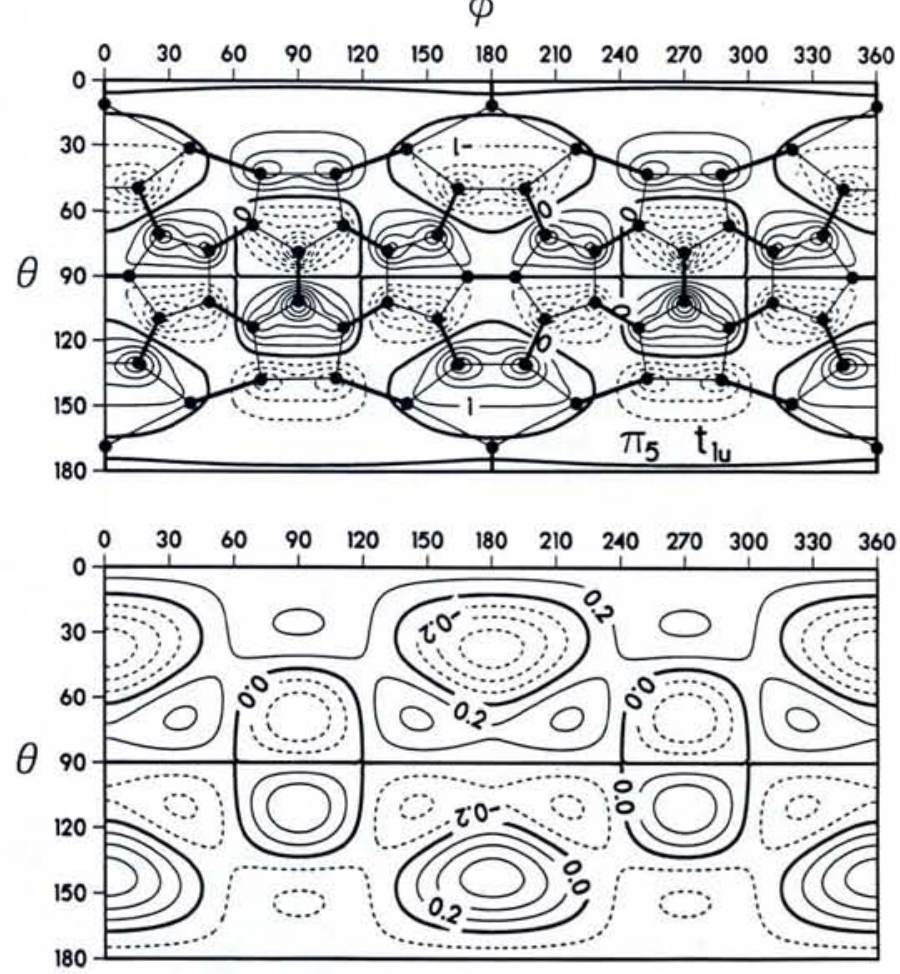

Fig. 3 - Contour plot of (a, upper) the wavefunction amplitude of the lowest unoccupied state of $C_{60}$ and $(b$, lower) a linear combination of spherical harmonics with $I=5$ that transforms with the $t_{1 u}$ representation of the $I_{h}$ icosahedral point group. The $C_{60}$ wavefunction is plotted at the surface of a sphere of $3.2 \AA$ radius as a function of azimuthal and polar angles. The dots and straight lines in (a) are the projection onto the sphere of the carbon atoms and bonding network, respectively. The thick contour lines emphasise the nodes of the functions. 
electron correlation effects that are not included in the traditional mean-field (e.g., local density) band structure calculations should have an important influence on the electronic properties. In particular, some of the alkali fullerides with stoichiometries lower than six and different from three could be Mott insulators. Note that the electronic system cannot be treated as an isolated narrow band, because the $\pi_{5}-t_{14}$ electrons have a strong spatial overlap with the remaining $\pi$ and $\sigma$ electrons. These electrons must be considered, for example, in the calculation of screening properties.

The many purely electronic mechanisms for superconductivity that have been proposed in the past for highly correlated electrons in a narrow conduction band may be responsible for superconductivity in the alkali fullerides at high temperatures. However, current experimental evidence indicates that superconductivity in alkali fullerides may be due to the traditional electron-phonon interaction. But the evidence is preliminary, and identifying the origin of the superconductivity in the alkali fullerides remains an important open question.

\section{Electron-phonon interaction}

The superconducting transition temperature $T_{c}$ of a material can be estimated from the (logarithmic) average phonon frequency $\omega_{\mathrm{log}}$, the strength of the electronphonon interaction, and the effective Coulomb interaction parameter $\mu^{*}$, using the Allen and Dynes version of the McMillan equation,

$$
\begin{aligned}
T_{c}= & \left(\hbar \omega_{\log } / 1.20 k_{\mathrm{B}}\right) \\
& \exp \left[-1.04(1+\lambda) /\left(\lambda-\mu^{*}-0.62 \mu^{*}\right)\right]
\end{aligned}
$$

Carbon-carbon bonds are strong and the carbon atomic mass is small, so the $C_{60}$ molecule has very high vibrational frequencies, with a maximum vibration energy of $\hbar \omega_{\max }=0.2 \mathrm{eV}$, and the value of $\hbar \omega_{\log } / k_{\mathrm{B}} \approx 1100 \mathrm{~K}$ is quite high. Using a typical value for metals of $\mu^{*} \approx 0.1$ and the observed $T_{\mathrm{c}}=18 \mathrm{~K}$, Eq. (1) gives $\lambda \approx$ 0.5 for $\mathrm{K}_{3} \mathrm{C}_{60}$ (for aluminum, $\lambda \approx 0.43$ and for lead, $\lambda \approx 1.55$ ). The high critical temperatures observed for alkali fullerides do not require an unusually strong electron-phonon interaction, but can be explained by the high molecular vibrational frequencies.

The usual microscopic expression for the electron-phonon interaction parameter is

$$
\lambda=2 N\left(E_{\mathrm{F}}\right) \bar{V},
$$

where $N\left(E_{\mathrm{F}}\right)$ is the density of states per spin at the Fermi energy and $\bar{V}$ is the average attractive electron-electron potential due to the electron-phonon interaction. The calculation of $\bar{V}$ requires an average over all the allowed scattering processes between two electron states by phonon absorption and emission. Recent theoretical estimates give $N\left(E_{\mathrm{F}}\right) \approx 8$ states per
$\mathrm{eV}$ per spin per $\mathrm{C}_{60}$, and $\bar{V} \approx 30 \mathrm{meV}$, hence $\lambda \approx 0.48$. This is the right order of magnitude considering the uncertainties in the theoretical estimates.

It has been observed that the critical temperature scales linearly with the lattice constant, $a$, of the alkali fullerides. If superconductivity is due to the interaction with the $\mathrm{C}_{60}$ vibrations, then $\bar{V}$ does not depend on the lattice constant, and $T_{c}$ depends on a through $N\left(E_{F}\right)$. The gradient of $\mathrm{d} T_{c} / \mathrm{d} a$ is consistent with current estimates of $\mathrm{d} N\left(E_{\mathrm{F}}\right) / \mathrm{da}$. This scaling of $T_{\mathrm{c}}$ with $a$ is one of the experimental observations that should be explained by any valid mechanism for superconductivity in the alkali fullerides.

The strongest evidence for a phonon coupling mechanism is the disappearance in metallic $\mathrm{K}_{3} \mathrm{C}_{60}$ of Raman lines that are observed in both insulating $\mathrm{C}_{60}$ and in $\mathrm{K}_{6} \mathrm{C}_{60}$. By a lucky coincidence, the $\mathrm{t}_{10}$ molecular orbitals belong to the vector representation of the point group $I_{h}$. Thus the vibrational modes which induce scattering between the conduction electrons of $\mathrm{K}_{3} \mathrm{C}_{60}$ are also those which are Raman active. One consequence of the electronphonon interaction is a reduction of the phonon lifetime, and consequent broadening of the phonon lines. The disappearance of the phonon lines in $\mathrm{K}_{3} \mathrm{C}_{60}$ can be explained by a broadening sufficiently large to make the lines indistinguishable from the background noise. One crucial experiment would be to search for a narrowing of the Raman lines below $T_{c}$ where the superconducting gap will restrict the phonon decay channels.

An objection which can be raised to the phonon mechanism of superconductivity is that alkali intercalated graphite, which shows many similarities with alkali fullerides, has much smaller critical temperatures. There is, however, a crucial difference between the two types of compounds: The $\pi$ electrons of $C_{60}$ are $\mathrm{sp}$ hybrids whereas the $\pi$ electrons of graphite are of pure atomic $p$ character. This $p$ character closes by symmetry many of the available electron-phonon channels in graphite. In particular, there is no coupling to the transverse phonon modes. With the strong dependence of $T_{c}$ on the electronphonon coupling $\lambda$, large differences in critical temperatures can be achieved if some of the scattering channels are closed. If the hypothesis that sp hybridization is important is confirmed, then it would be the curvature of the bonding network which is responsible for the high $T_{c}$ in the alkali fullerides.

\section{Conclusions}

The $\mathrm{C}_{60}$ and $\mathrm{A}_{n} \mathrm{C}_{60}$ crystals have electronic and vibrational structures which can be simply understood once we consider their molecular character and the high symmetry and quasi-spherical shape of $\mathrm{C}_{60}$ molecules. There remains much to learn about superconductivity in highly correlated systems from the study of these beautiful compounds.

\section{FURTHER READING}

The field was recently reviewed by R.F. Curl and R.E. Smalley in Scientific American (October 1991) and by D.R. Huffman in Physics Today (November 1991). For an extensive list of the $C_{60}$ literature, send an e-mail message to the "Bucky News Service", bucky@sol1.Irsm.upenn.edu, with the single word "INTRO" in the first line.

\title{
UNIVERSITY OF DORTMUND
}

The Faculty (Fachbereich) of Physics invites applications for a

\section{Full-Professorship (Universitätsprofessor C4)}

\author{
(successor of Prof. Dr. h.c. Ulrich Bonse)
}

\section{for Experimental Physics}

The position is expected to be available from October 1st, 1993.

Applicants should show a successful research record in the field of experimental solid state physics. Their scientific activities should be concentrated in applications of Synchrotron radiation and/or neutrons in solid state physics. Scientific collaboration with existing experimental and theoretical groups of the Faculty is encouraged.

The appointee is expected to participate actively in research, teaching and administration of the Faculty and to contribute to the Graduate Schools Program "Solid State Spectroscopy". A close cooperation with the Institute of Accelerator Physics and Synchrotron Radiation (DELTA project) is requested.

Applicants must satisfy the legal requirements of the Land Nordrhein Westfalen for the above appointment, namely Habilitation or the equivalent scientific merits or degrees.

Applicants should send their curriculum vitae, a summary of their scientific career including teaching experience, and a list of publications by the 15th of April to:

Dekan des Fachbereichs Physik der Universität Dortmund, Postfach 5005 00, W-4600 Dortmund 50, Germany. 